\title{
Review Article \\ Relationship of Obstructive Sleep Apnoea with Diabetic Retinopathy: A Meta-Analysis
}

\author{
Zhenliu Zhu, ${ }^{1}$ Fengying Zhang, ${ }^{2}$ Yunxia Liu, ${ }^{3}$ Shuqin Yang, ${ }^{4}$ Chunting Li, ${ }^{5}$ \\ Qingliang Niu, ${ }^{6}$ and Jinming Niu ${ }^{2}$ \\ ${ }^{1}$ Ophthalmology Department, Weifang Traditional Chinese Hospital, 1055 Weizhou Road, Kuiwen District, Weifang 261000, China \\ ${ }^{2}$ Otorhinolaryngological Department, Weifang Traditional Chinese Hospital, 1055 Weizhou Road, Kuiwen District, \\ Weifang 261000, China \\ ${ }^{3}$ Outpatient Operating Room, Weifang Traditional Chinese Hospital, 1055 Weizhou Road, Kuiwen District, Weifang 261000, China \\ ${ }^{4}$ Otorhinolaryngological Department, Weifang People’s Hospital, 151 Guangwen Street, Kuiwen District, Weifang 261000, China \\ ${ }^{5}$ Yantai KangAi Eye Hospital, 26 Zhichu Road, Zhifu District, Yantai 264000, China \\ ${ }^{6}$ Imaging Department, Weifang Traditional Chinese Hospital, 1055 Weizhou Road, Kuiwen District, Weifang 261000, China
}

Correspondence should be addressed to Fengying Zhang; ym00223@126.com

Received 9 June 2017; Accepted 4 October 2017; Published 5 November 2017

Academic Editor: Jan Plzak

Copyright (C) 2017 Zhenliu Zhu et al. This is an open access article distributed under the Creative Commons Attribution License, which permits unrestricted use, distribution, and reproduction in any medium, provided the original work is properly cited.

\begin{abstract}
Until now, the relationship of obstructive sleep apnoea (OSA) with diabetic retinopathy (DR) was controversial. This meta-analysis was performed to obtain definitive conclusion on this topic. Relevant articles were searched on databases of Pubmed, Google Scholar, and Chinese National Knowledge Infrastructure (CNKI). The articles were selected according to inclusion and exclusion criteria. Odds ratio (OR) with $95 \%$ confidence interval (CI) was used to evaluate the relationship of OSA with risk of DR. $I^{2}$ and $P$ value were used to assess the presence of heterogeneity. $I^{2} \geq 50 \%$ or $P<0.05$ indicated significant heterogeneity. Sensitivity analysis was performed to evaluate the robustness of pooled results. Begg's funnel plot and Egger's regression analysis were adopted to assess publication bias. 6 eligible studies were selected in the present meta-analysis. The pooled results indicated that OSA was significantly associated with increased risk of DR $(\mathrm{OR}=2.01,95 \% \mathrm{CI}=1.49-2.72)$. Subgroup analysis based on type of diabetes mellitus suggested that OSA was related to DR in both Type 1 and Type 2 diabetes mellitus. Sensitivity analysis demonstrated that pooled results were robust. No significant publication bias was observed $(P=0.128)$. The results indicate that OSA is related to increased risk of DR.
\end{abstract}

\section{Introduction}

Obstructive sleep apnoea (OSA) is a disease featured by hypopnoea and/or apnoea during sleep that is commonly brought about by airway arousals and obstruction [1]. It affects $2 \%$ of women and $4 \%$ of men [2]. Obesity is a common risk factor for OSA and neck circumference is tightly correlated with the severity of OSA [3]. It has been estimated that about $75 \%$ of individuals with BMI $>40 \mathrm{~kg} / \mathrm{m}^{2}$ have OSA [4]. OSA patients usually show extensive daytime sleepiness, cardiovascular disease, mood changes, and cognitive dysfunction [5]. In addition, OSA patients are more likely to develop metabolic syndrome, insulin resistance, vascular disease, and diabetes mellitus [6-9]. Moderate to severe OSA has been demonstrated to be related to enhanced risk of diabetes mellitus [10].

In addition, episodes of hypoxemia in OSA may be correlated with increased inflammatory mediators [11]. For these patients with diabetes mellitus, the levels of inflammatory mediators are also increased [12], which is significantly associated with diabetic microvascular complications, such as diabetic maculopathy and diabetic retinopathy (DR) [13]. It has been demonstrated that OSA in the patients with diabetic mellitus may be related with much more advanced diabetic maculopathy and more DR cases, which suggests the significant relationship of OSA with DR $[14,15]$.

Although extensive attention has been paid to the relationship of OSA with DR, no consistent conclusion has 
TABLE 1: Main information about the included studies.

\begin{tabular}{|c|c|c|c|c|c|}
\hline \multirow{2}{*}{ Author } & \multirow{2}{*}{ Year } & \multirow{2}{*}{ Country } & \multicolumn{2}{|c|}{ Sample size, $n$} & \multirow{2}{*}{ Type of DM } \\
\hline & & & OSA+ & OSA- & \\
\hline Banerjee & 2013 & United Kingdom & 46 & 47 & Type 2 \\
\hline Manin & 2014 & France & 31 & 36 & Type 1 \\
\hline Storgaard & 2014 & Denmark & 72 & 108 & Type 2 \\
\hline West & 2010 & United Kingdom & 28 & 90 & Type 2 \\
\hline Zhang & 2015 & China & 310 & 162 & Type 2 \\
\hline Zhang & 2016 & China & 121 & 41 & Type 2 \\
\hline
\end{tabular}

been obtained. The present meta-analysis was performed to extract a definitive conclusion on this issue. The outcome may contribute to screening OSA patients with high risk of DR and providing preventive strategy for these individuals.

\section{Materials and Methods}

2.1. Search Strategy. F. Zhang and Z. Zhu were responsible for searching articles on databases of Pubmed, Google Scholar, and Chinese National Knowledge Infrastructure (CNKI). The date was limited to June 2017. The terms used in the search were as follows: obstructive sleep apnoea OR OSA; diabetic retinopathy OR DR. Only the articles in English and Chinese were considered. We usually selected the recently published articles when we found two or more articles with same population. Meanwhile, we reviewed the references of obtained articles for relevant articles.

2.2. Inclusion and Exclusion Criteria. The articles were selected according to the inclusion and exclusion criteria. The inclusion criteria were listed as follows: (a) case-control studies; (b) the articles investigating the relationship of OSA and DR; (c) the articles providing the frequency of DR in case $(\mathrm{OSA}+)$ and control (OSA-) groups. Review articles and case reports would be excluded from the analysis.

2.3. Data Extraction. Data extraction was independently performed by two authors (Y. Liu and S. Yang). The controversial issues would be solved with a discussion with the third author (F. Zhang). They usually reviewed the data together and had discussion until consistent opinion was reached. The data were name of first author, year of publication, country, sample size, type of diabetes mellitus, and frequency of DR in each group.

2.4. Statistical Analysis. All the analysis was performed in Stata 12.0 software. Odds ratio (OR) with corresponding 95\% confidence interval (CI) was calculated to evaluate the relationship of OSA with DR. $I^{2}$ and $P$ value were used to assess the existence of heterogeneity. $I^{2} \geq 50 \%$ or $P<0.05$ indicated significant heterogeneity. The data was pooled with the random-effects model if the heterogeneity presented; otherwise, it was pooled with the fixed-effects model. Sensitivity analysis was performed to evaluate the robustness of pooled outcomes. Begg's funnel plot and Egger's regression analysis were adopted to detect potential publication bias.

\section{Results}

3.1. Article Selection Process. After rough search, a total of 132 relevant articles were obtained. Then the titles and abstracts were screened. Subsequently, 96 articles were removed for review articles $(n=33)$, investigating prevalence of OSA in patients with diabetes mellitus $(n=27)$ and analyzing the relationship of OSA with other complications of diabetes mellitus $(n=36)$. For the remaining 36 articles, 30 articles were removed for unavailable data $(n=17)$ and analyzing the relationship of parameters of OSA with DR $(n=13)$. At last, 6 eligible studies were included [14,16-20]. The selection process was shown in Figure 1. The detailed information of each study was listed in Table 1.

3.2. Relationship of Obstructive Sleep Apnoea with Diabetic Retinopathy. The outcome about the relationship of OSA with DR was shown in Figure 2. The pooled data was calculated with the fixed-effects model. It suggested that OSA was significantly related to increased risk of DR $(\mathrm{OR}=2.01$, $95 \%$ CI $=1.49-2.72$ ). Subgroup analysis by type of diabetes mellitus was also performed. OSA was associated with the risk of DR among both of patients with Type 1 and Type 2 diabetes mellitus.

3.3. Sensitivity Analysis and Publication Bias Detection. Sensitivity analysis was performed to assess the robustness of pooled results. It demonstrated that the pooled results were robust. Publication bias was detected via Begg's funnel plot (Figure 3) and Egger's regression analysis. In the present meta-analysis, no obvious publication bias was observed $(P=$ 0.128).

\section{Discussion}

The present meta-analysis suggested that OSA was significantly associated with increased risk of DR. The outcome was observed in both Type 1 and Type 2 diabetes mellitus. In the analysis, no obvious heterogeneity and publication bias were observed. The pooled results were credible.

The association between OSA and Type 2 diabetes mellitus has been well defined. Onset of OSA is correlated with increased insulin resistance/glucose intolerance. Meanwhile, diabetes mellitus may aggravate sleep-disordered breathing due to autonomic neuropathy [21]. It is also reported that insulin resistance could predict the onset of OSA. Therefore, 


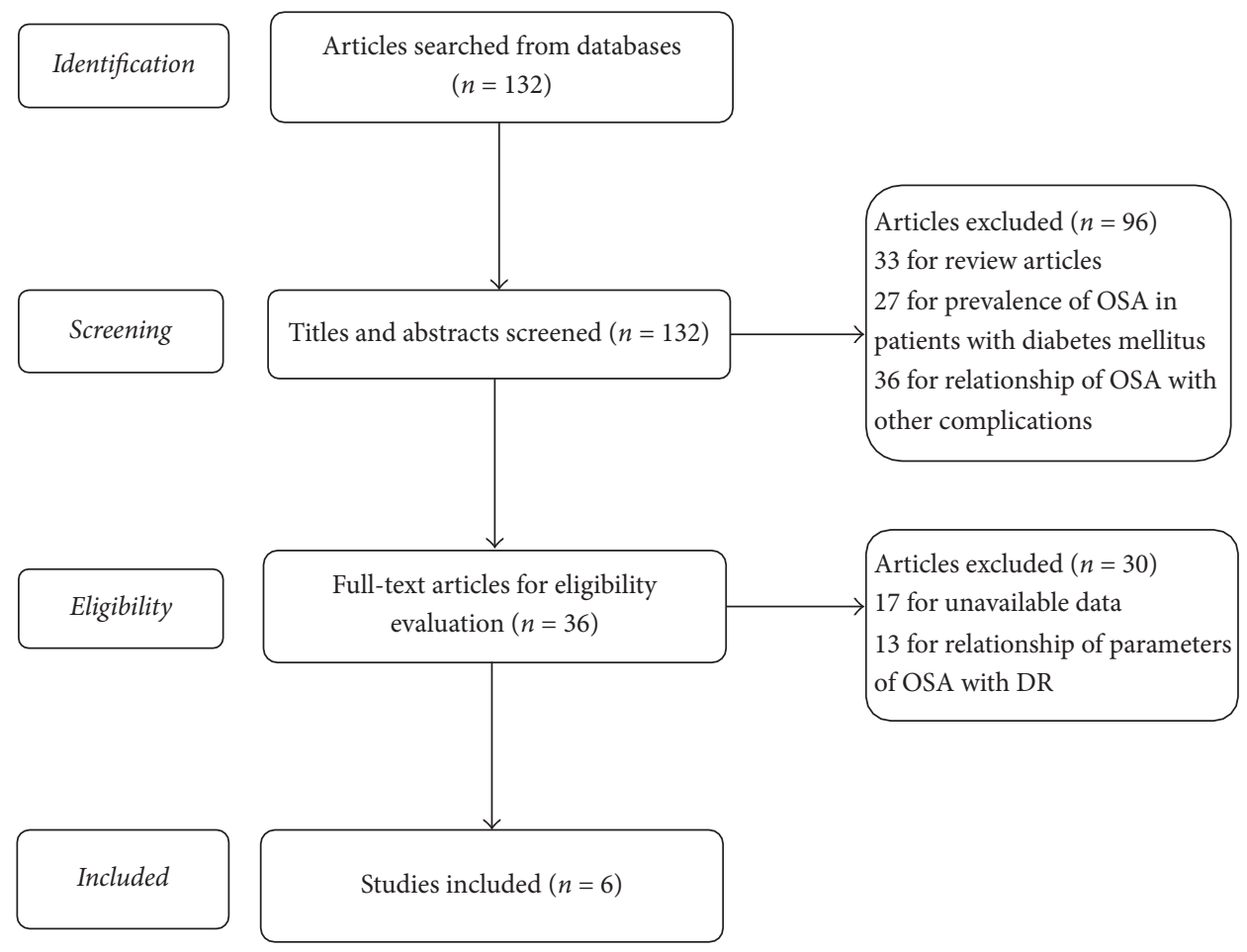

FIGURE 1: Selection process of eligible articles. 6 eligible articles were selected.

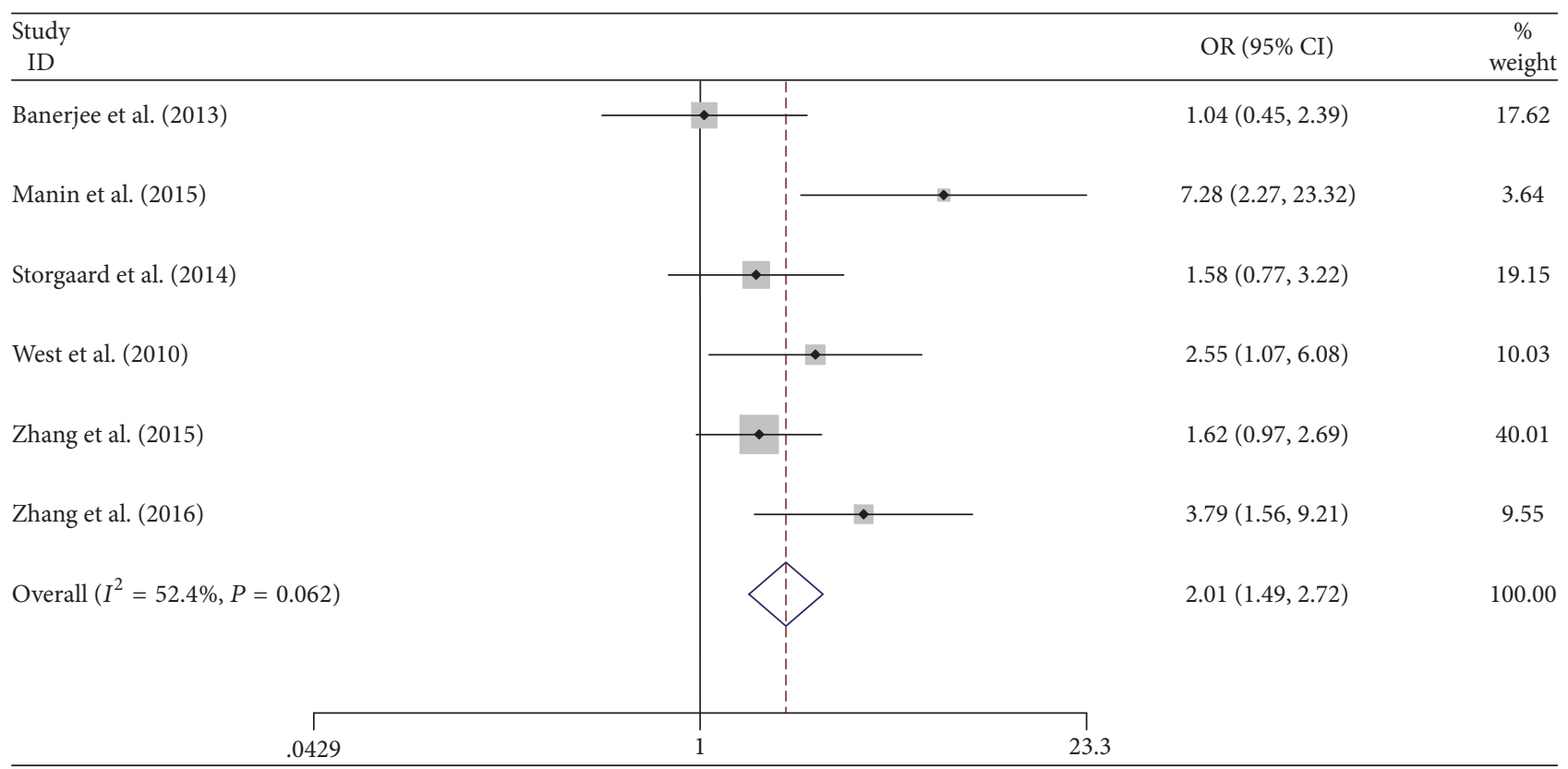

FIGURE 2: Relationship of OSA with DR. The results indicated that OSA was significantly associated with risk of DR $(\mathrm{OR}=2.01,95 \% \mathrm{CI}=$ $1.49-2.72)$.

the association between insulin resistance and OSA seems to be bidirectional [22]. In addition, Type 2 diabetes mellitus shares same risk factor with OSA, such as obesity $[23,24]$. Obesity could cause insulin resistance/glucose intolerance. Meanwhile, it appears as an important risk factor of OSA. It has been estimated that prevalence of OSA ranges from $17 \%$ to $86 \%$ in patients with Type 2 diabetes mellitus in the Japan, US, and Hong Kong [25-27].

DR is one of microvascular complications of diabetes mellitus, which presents as a main cause of visual impairment among working-age population. Thus, early diagnosis of DR in the patients with diabetes mellitus is vital. However, the 


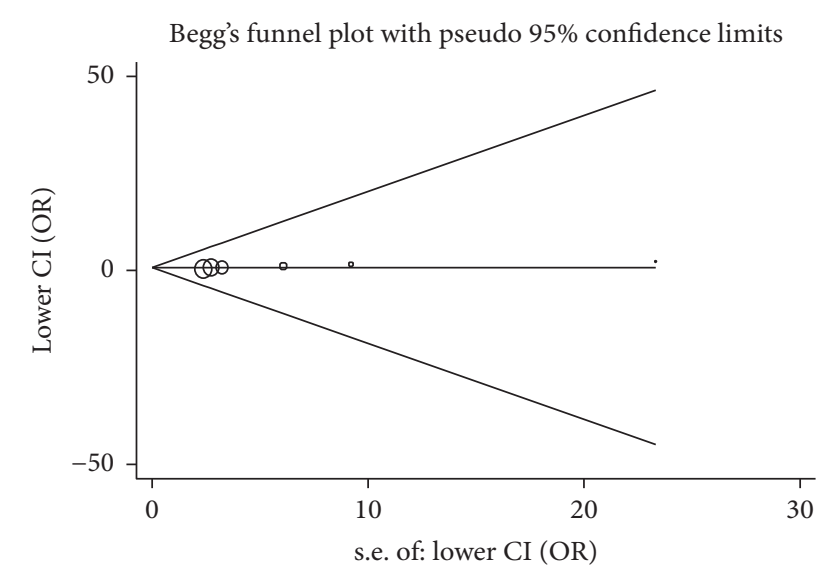

Figure 3: Begg's funnel plot. No significant publication bias was observed $(P=0.128)$.

pathogenesis of DR is not fully explained up to now $[28,29]$. Known risk factors of DR include older age, longer duration of diabetes mellitus, cardiovascular events, and aging [30, 31]. Alcohol intake is also regarded as one risk factor of DR [32]. The recent studies found significant relationship of OSA with risk of DR.

About the relationship of OSA with DR, there was no consistent conclusion until now. On this topic, Leong et al. also initiated a meta-analysis. Their analyses suggested that minimum oxygen saturation showed impact on DR $(\mathrm{OR}=0.91,95 \% \mathrm{CI}=0.87-0.95)$ [33]. The cross-sectional study by Manin et al. reported that OSA was correlated with the development of macrovascular complications and retinopathy [17]. In another study by West et al., retinopathy and maculopathy scores were found to be much worse in the OSA group. Multiple regression analysis suggested that OSA was an independent predictor of retinopathy $\left(R^{2}=\right.$ $0.19, P<0.0001)$ [14]. Banerjee et al. investigated the potential relationship of OSA and DR among patients with severe obesity and concluded that there was no significant relationship of OSA and diabetic retinal complications [16].

Our analysis was based on 6 eligible articles and the results were credible. However, the sample size was relatively small, which may affect the accuracy of results. Moreover, much larger-scale research, such as multiple-centre study, should be performed to confirm the results. In addition, the pathogenesis of DR involves many factors; only OSA was analyzed in the present meta-analysis, which cannot fully explain the developmental mechanism of DR. More comprehensive analysis will contribute to uncovering the pathogenesis of DR and more factors should be considered in the future analysis. Meanwhile, most studies analyzed Type 2 diabetes mellitus and only one study involved Type 1 diabetes mellitus. The relationship of OSA with DR in patients with Type 1 diabetes mellitus should be verified.

\section{Conclusion}

In conclusion, the presence of OSA is related to increased risk of DR. The conclusion is helpful for screening the individuals with high risk of DR among the patients with diabetes mellitus. It is significant for clinical physicians.

\section{Disclosure}

Zhenliu Zhu and Fengying Zhang are co-first authors.

\section{Conflicts of Interest}

All authors declared that there were no conflicts of interest.

\section{Authors' Contributions}

Zhenliu Zhu and Fengying Zhang contributed equally to this work.

\section{Acknowledgments}

The authors of this paper are indebted to the primary studies' authors.

\section{References}

[1] L. J. Epstein, D. Kristo, P. J. Strollo Jr. et al., "Clinical guideline for the evaluation, management and long-term care of obstructive sleep apnea in adults," Journal of Clinical Sleep Medicine, vol. 5, no. 3, pp. 263-276, 2009.

[2] T. Young, M. Palta, J. Dempsey, J. Skatrud, S. Weber, and S. Badr, "The occurrence of sleep-disordered breathing among middleaged adults," The New England Journal of Medicine, vol. 328, no. 17, pp. 1230-1235, 1993.

[3] R. J. O. Davies, N. J. Ali, and J. R. Stradling, "Neck circumference and other clinical features in the diagnosis of the obstructive sleep apnoea syndrome," Thorax, vol. 47, no. 2, pp. 101-105, 1992.

[4] P. P. Lopez, B. Stefan, C. I. Schulman, and P. M. Byers, "Prevalence of sleep apnea in morbidly obese patients who presented for weight loss surgery evaluation: More evidence for routine screening for obstructive sleep apnea before weight loss surgery," The American Surgeon, vol. 74, no. 9, pp. 834-838, 2008.

[5] A. I. Sánchez, P. Martínez, E. Miró, W. A. Bardwell, and G. Buela-Casal, "CPAP and behavioral therapies in patients with obstructive sleep apnea: effects on daytime sleepiness, mood, and cognitive function," Sleep Medicine Reviews, vol. 13, no. 3, pp. 223-233, 2009.

[6] L. F. Drager, H. F. Lopes, C. Maki-Nunes et al., "The impact of obstructive sleep apnea on metabolic and inflammatory markers in consecutive patients with metabolic syndrome," PLoS ONE, vol. 5, no. 8, Article ID e12065, 2010.

[7] P. E. Peppard, T. Young, M. Palta, J. Dempsey, and J. Skatrud, "Longitudinal study of moderate weight change and sleepdisordered breathing," The Journal of the American Medical Association, vol. 284, no. 23, pp. 3015-3021, 2000.

[8] A. Shankar, P. E. Peppard, T. Young, B. E. K. Klein, R. Klein, and F. J. Nieto, "Sleep-disordered breathing and retinal microvascular diameter," Atherosclerosis, vol. 226, no. 1, pp. 124128, 2013.

[9] H. E. Resnick, S. Redline, E. Shahar et al., "Diabetes and sleep disturbances: findings from the Sleep Heart Health Study," Diabetes Care, vol. 26, no. 3, pp. 702-709, 2003. 
[10] X. Wang, Y. Bi, Q. Zhang, and F. Pan, “Obstructive sleep apnoea and the risk of type 2 diabetes: A meta-analysis of prospective cohort studies," Respirology, vol. 18, no. 1, pp. 140-146, 2013.

[11] R. Schulz, C. Hummel, S. Heinemann, W. Seeger, and F. Grimminger, "Serum levels of vascular endothelial growth factor are elevated in patients with obstructive sleep apnea and severe nighttime hypoxia," American Journal of Respiratory and Critical Care Medicine, vol. 165, no. 1, pp. 67-70, 2002.

[12] L. P. Aiello, R. L. Avery, P. G. Arrigg et al., "Vascular endothelial growth factor in ocular fluid of patients with diabetic retinopathy and other retinal disorders," The New England Journal of Medicine, vol. 331, no. 22, pp. 1480-1487, 1994.

[13] C. Bunce and R. Wormald, "Leading causes of certification for blindness and partial sight in England \& Wales," BMC Public Health, vol. 6, p. 58, 2006.

[14] S. D. West, D. C. Groves, H. J. Lipinski et al., "The prevalence of retinopathy in men with Type 2 diabetes and obstructive sleep apnoea," Diabetic Medicine, vol. 27, no. 4, pp. 423-430, 2010.

[15] T. Shiba, Y. Sato, and M. Takahashi, "Relationship Between Diabetic Retinopathy and Sleep-Disordered Breathing," American Journal of Ophthalmology, vol. 147, no. 6, pp. 1017-1021, 2009.

[16] D. Banerjee, W. B. Leong, T. Arora et al., "The potential association between obstructive sleep apnea and diabetic retinopathy in severe obesity - The role of hypoxemia," PLoS ONE, vol. 8, no. 11, Article ID e79521, 2013.

[17] G. Manin, A. Pons, P. Baltzinger et al., "Obstructive sleep apnoea in people with Type 1 diabetes: Prevalence and association with micro- and macrovascular complications," Diabetic Medicine, vol. 32, no. 1, pp. 90-96, 2015.

[18] H. Storgaard, B. Mortensen, T. Almdal, M. Laub, and L. Tarnow, "At least one in three people with type 2 diabetes mellitus referred to a diabetes centre has symptomatic obstructive sleep apnoea," Diabetic Medicine, vol. 31, no. 11, pp. 1460-1467, 2014.

[19] R. Zhang, X. Guo, L. Guo, J. Lu, X. Zhou, and L. Ji, "Prevalence and associated factors of obstructive sleep apnea in hospitalized patients with type 2 diabetes in Beijing, China," Journal of Diabetes, vol. 7, no. 1, pp. 16-23, 2015.

[20] R. Z. Zhang, F. Wang, and S. Q. Wei, "Influence of OSAS on the blood glucose and related complications of the patients with type 2 diabetes mellitus," Journal of Bethune Medical Science, vol. 14, no. 5, pp. 585-587, 2016 (Chinese).

[21] P. Bottini, S. Redolfi, M. L. Dottorini, and C. Tantucci, "Autonomic neuropathy increases the risk of obstructive sleep apnea in obese diabetics," Respiration, vol. 75, no. 3, pp. 265-271, 2008.

[22] B. Balkau, S. Vol, S. Loko et al., "High baseline insulin levels associated with 6-year incident observed sleep apnea," Diabetes Care, vol. 33, no. 5, pp. 1044-1049, 2010.

[23] S. P. Patil, H. Schneider, A. R. Schwartz, and P. L. Smith, "Adult obstructive sleep apnea: pathophysiology and diagnosis," CHEST, vol. 132, no. 1, pp. 325-337, 2007.

[24] H. Y. Sun, F. D. Ke, and J. S. Wen, "Prevalence of diabetes among men and women in China [5]," The New England Journal of Medicine, vol. 362, no. 25, pp. 2425-2426, 2010.

[25] D. C. L. Lam, M. M. S. Lui, J. C. M. Lam, L. H. Y. Ong, K. S. L. Lam, and M. S. M. Ip, "Prevalence and recognition of obstructive sleep apnea in Chinese patients with type 2 diabetes mellitus," CHEST, vol. 138, no. 5, pp. 1101-1107, 2010.

[26] D. Einhorn, D. A. Stewart, M. K. Erman, N. Gordon, A. Philis-Tsimikas, and E. Casal, "Prevalence of sleep apnea in a population of adults with type 2 diabetes mellitus," Endocrine Practice, vol. 13, no. 4, pp. 355-362, 2007.
[27] S. Kashine, K. Kishida, T. Funahashi et al., "Characteristics of sleep-disordered breathing in Japanese patients with type 2 diabetes mellitus," Metabolism - Clinical and Experimental, vol. 59, no. 5, pp. 690-696, 2010.

[28] C.-J. Shih, H.-T. Chen, S.-C. Kuo et al., "Comparative effectiveness of angiotensin-converting-enzyme inhibitors and angiotensin II receptor blockers in patients with type 2 diabetes and retinopathy," Canadian Medical Association Journal, vol. 188, no. 8, pp. E148-E157, 2016.

[29] D. J. Pieramici, P.-W. Wang, B. Ding, and S. Gune, "Visual and Anatomic Outcomes in Patients with Diabetic Macular Edema with Limited Initial Anatomic Response to Ranibizumab in RIDE and RISE Presented in part at: American Academy of Ophthalmology Annual Meeting, October 2014, Chicago, Illinois; And 38th Macula Society Annual Meeting, February 2015, Scottsdale, Arizona.," Ophthalmology, vol. 123, no. 6, pp. 1345-1350, 2016.

[30] U. Alam, V. Arul-Devah, S. Javed, and R. A. Malik, "Vitamin D and Diabetic Complications: True or False Prophet?" Diabetes Therapy, vol. 7, no. 1, pp. 11-26, 2016.

[31] R. Ahmed, S. Khalil, and M. Al-Qahtani, "Diabetic retinopathy and the associated risk factors in diabetes type 2 patients in Abha, Saudi Arabia," Journal of Family and Community Medicine (JFCM), vol. 23, no. 1, pp. 18-24, 2016.

[32] E. Martín-Merino, J. Fortuny, E. Rivero-Ferrer, M. Lind, and L. A. Garcia-Rodriguez, "Risk factors for diabetic retinopathy in people with Type 2 diabetes: A case-control study in a UK primary care setting," Primary Care Diabetes, vol. 10, no. 4, pp. 300-308, 2016.

[33] W. B. Leong, F. Jadhakhan, S. Taheri, Y. F. Chen, P. Adab, and G. N. Thomas, "Effect of obstructive sleep apnoea on diabetic retinopathy and maculopathy: A systematic review and metaanalysis," Diabetic Medicine, vol. 33, no. 2, pp. 158-168, 2016. 


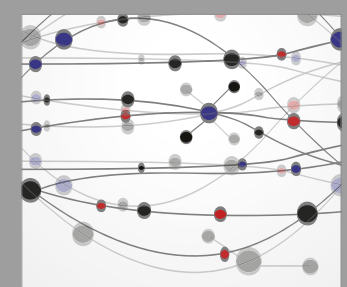

The Scientific World Journal
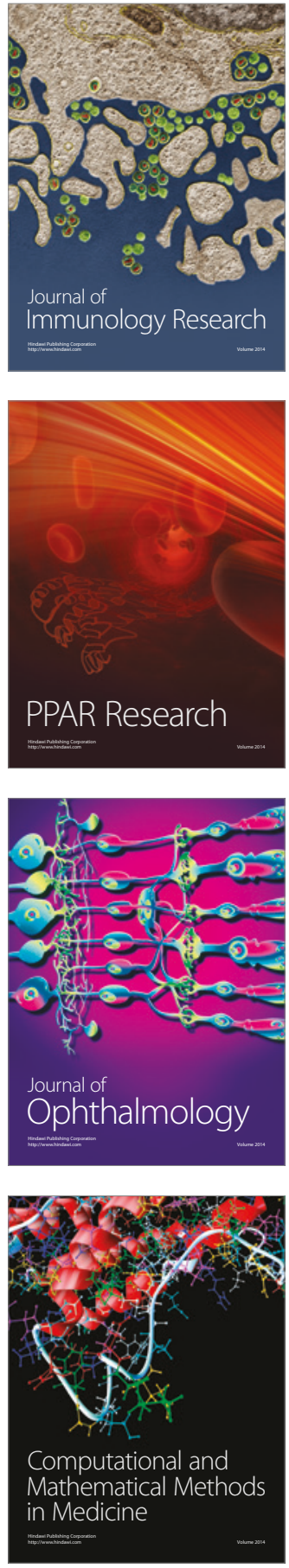

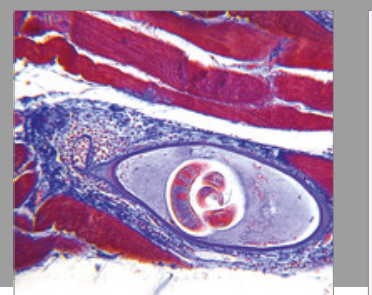

Gastroenterology Research and Practice
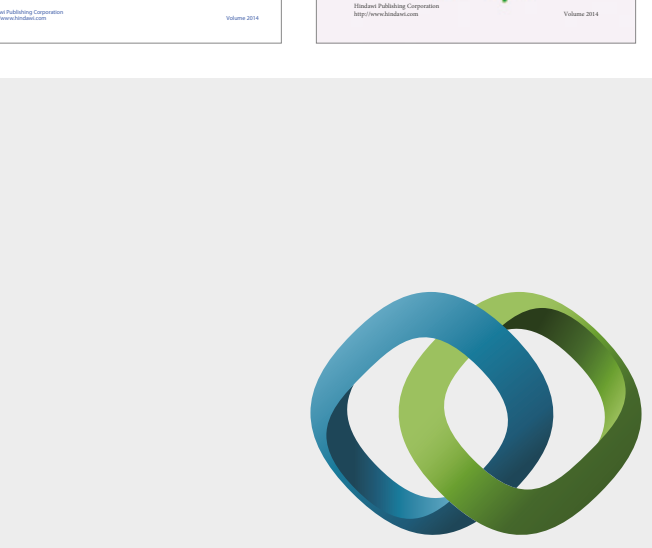

\section{Hindawi}

Submit your manuscripts at

https://www.hindawi.com
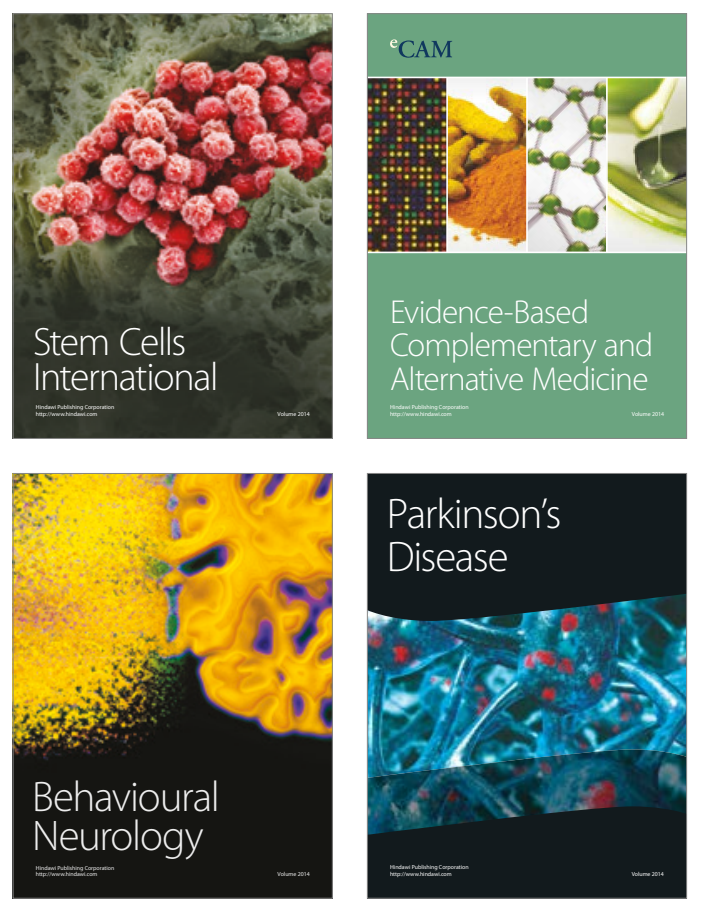
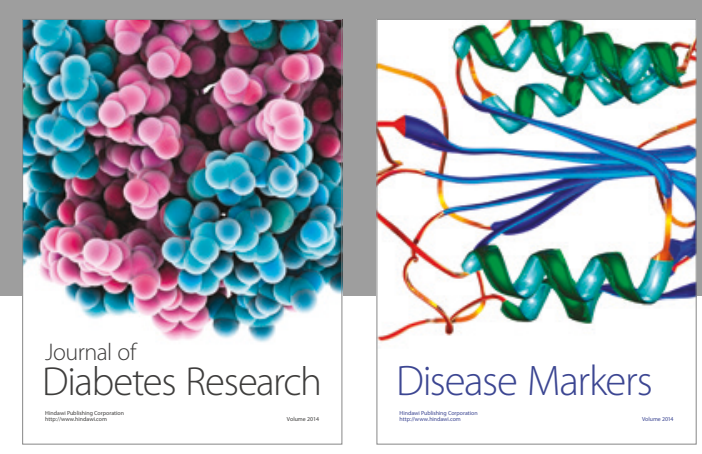

Disease Markers
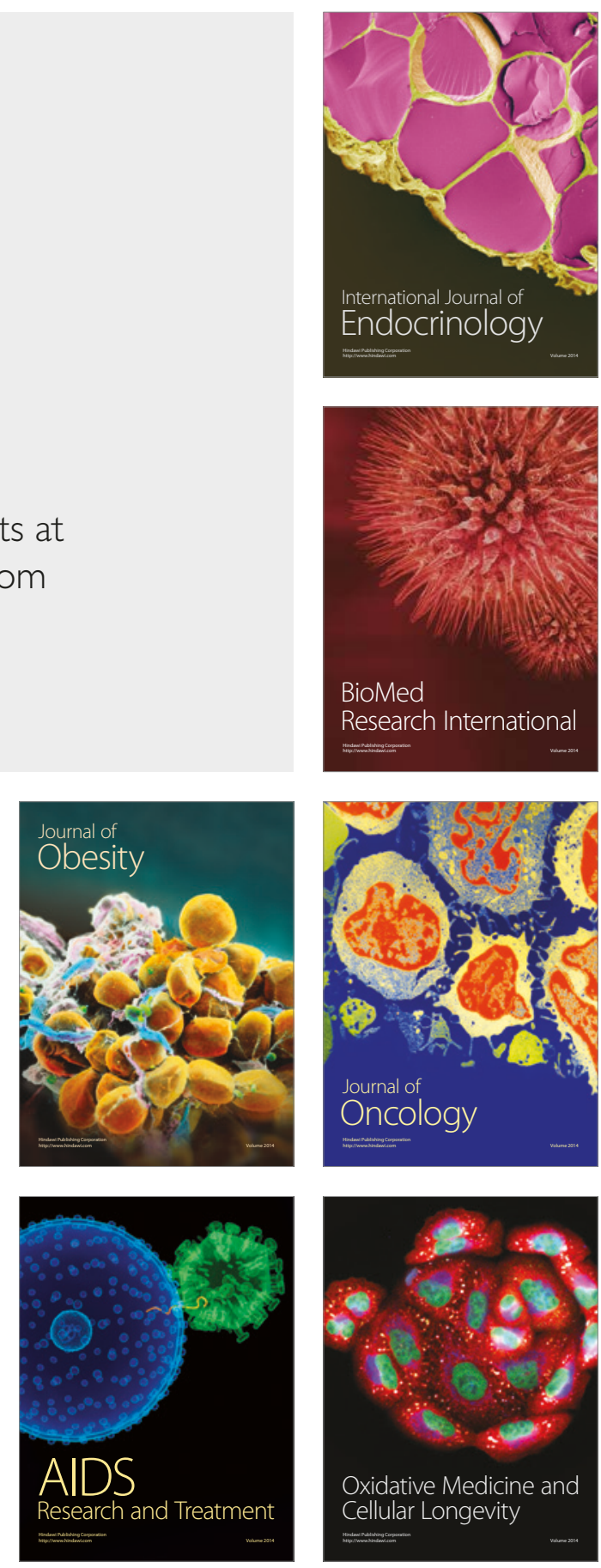